\title{
EL COMPONENTE CULTURAL Y LOS ESTEREOTIPOS EN EL MANUAL CHINO PARA EL ESTUDIO DEL ESPAÑOL EN CONTEXTOS UNIVERSITARIOS
}

\author{
María Macarro Maté ${ }^{1}$ \\ Universidad Camilo José Cela. Madrid, España \\ Mayra Martínez Avidad \\ Universidad Camilo José Cela. Madrid, España
}

\begin{abstract}
Resumen. No es posible enseñar ni aprender un idioma sin hacer constante referencia a la cultura de sus hablantes, ya que el uso del lenguaje implica en sí mismo una serie de códigos de conducta y una simbología. El componente cultural es por tanto, una parte fundamental en el estudio y dominio de cualquier lengua. El uso correcto de lengua requiere, además del conocimiento gramatical y lingüístico, un preciso conocimiento cultural que permita a los hablantes interpretar con precisión el contenido de la comunicación. En esta investigación analizamos el manual "Español Moderno", el libro de texto que se usa en las universidades chinas desde los años 60 para el aprendizaje del español. El objetivo es determinar la presencia y exactitud de los contenidos sobre la cultura española dentro del manual, con vistas a determinar si desde el mismo se hace una buena aproximación a esta parte del aprendizaje de la lengua. Para ello hemos utilizado una metodología de análisis de contenido usando como referencia el apartado Saberes y Comportamientos culturales del Plan Curricular del Instituto Cervantes. Además, hacemos un análisis de los estereotipos sobre la cultura española presentes en el manual. Los resultados del estudio reflejan que los contenidos que se enseñan sobre la cultura española entre los alumnos universitarios chinos a través del manual son insuficientes y en ocasiones estereotipados.
\end{abstract}

Palabras clave: Español moderno, China, componente cultural, enseñanza de ELE, estereotipos.

\section{THE CULTURAL COMPONENT AND STEREOTYPES IN THE CHINESE BOOK "ESPAÑOL MODERNO"}

\begin{abstract}
It is not possible to teach or learn a language without constant reference to the culture of its speakers, since the use of language itself involves codes of conduct and symbology. The cultural component is therefore a fundamental part in the study and mastery of any language. The correct use of any language requires, in addition to grammatical and linguistic knowledge, cultural knowledge precise to enable speakers interpret the content of the communication. In this research, we analyzed the manual "Español Moderno" is a textbook used in Chinese universities since the 60s for learning Spanish. The objective is to determine the presence and accuracy of the contents of the Spanish culture within the manual, in order to determine if it is used for a good approximation to this part of language learning. To check this in the manual, we have used a content analysis methodology, using as a reference, the section Saberes y comportamientos culturales into the Cervantes Institute Curricular Plan. In addition, we analyze stereotypes about Spanish culture. The study results shows us that the contents taught about the
\end{abstract}

\footnotetext{
${ }^{1}$ Correspondencia: Gabriel Travé González. Campus “El Carmen” Avenida de las Fuerzas Armadas C.P. 21071 Huelva. gabriel.trave@dedu.uhu.es
} 
Spanish culture among Chinese university students through the manual, are insufficient and sometimes stereotyped.

Keywords: Español Moderno, China, cultural component, ELE teaching, stereotypes.

\title{
O COMPONENTE CULTURAL E OS ESTEREÓTIPOS NO MANUAL CHINÉS “ESPAÑOL MODERNO"
}

\begin{abstract}
Resumo. Você não pode ensinar ou aprender uma língua sem referência constante à cultura de seus altofalantes, uma vez que o uso da própria linguagem envolve uma série de códigos de conduta e simbologia. O componente cultural é, portanto, uma parte fundamental no estudo e domínio de qualquer língua. O uso correto da linguagem requer, além de conhecimentos gramaticais e linguística, conhecimento cultural precisa para permitir falantes interpretar com precisão o conteúdo da comunicação. Nesta pesquisa analisou o manual do livro "Modern espanhol" usado em universidades chinesas desde os anos 60 para aprender espanhol. O objectivo é determinar a presença e precisão do conteúdo da cultura espanhola dentro do manual, a fim de determinar se uma boa aproximação a esta parte de aprendizagem de línguas a partir dele. Então, nós usamos uma metodologia de análise de conteúdo utilizando como referência o cultural Comportamentos Saberes Instituto Cervantes Plano Curricular e seção. Além disso, analisamos os estereótipos sobre a cultura espanhola no manual. Os resultados do estudo mostram que os conteúdos ensinados sobre a cultura espanhola entre estudantes universitários chineses através do manual são insuficientes e, por vezes estereotipados.
\end{abstract}

Palavras-chave: Español Moderno, chineses, componente cultural, ensinando ELE, estereótipos.

\section{Introducción}

Desde que en 1952, la universidad Estudios Extranjeros de Pekín, popularmente conocida como Beiwai, abriera el primer departamento de español en 1952, la demanda de este idioma en China no ha parado de crecer. Aunque posicionada detrás del inglés o de idiomas más cercanos a China geográficamente, el español se va consolidando como un idioma cada vez más importante en este país. Más de 2.000 hispanistas han salido solo de la universidad de Beiwai (datos de 2012) y actualmente el español se enseña en más de 100 universidades chinas en distintos grados: en 60 como licenciatura, en 20 como diplomatura y en el resto como ELE (Fachal, 2012).

La presencia desde el año 2006 del Instituto Cervantes (IC) de Pekín, también ha favorecido el aprendizaje de esta lengua. Actualmente el IC celebra cinco convocatorias al año de los exámenes de DELE (Diploma de español como lengua extranjera) y está previsto fijar un acuerdo con la autoridad nacional en materia de exámenes de lenguas extranjeras, para introducir el DELE en más puntos del país, ya que hasta la fecha solamente se celebran en Pekín, Shanghái y Hong Kong (Ministerio de Educación Cultura y Deporte, 2014). 
El Ministerio de Educación Cultura y deporte de España, presente en China desde 2005, a través de su Consejería de educación de Pekín, ha llevado a cabo, desde entonces, un registro de estudiantes de español. Según sus datos, en el año 2005, 4.231 alumnos estudiaban español en todo el país ese año, una cifra que se ha incrementado hasta los 31.154 que lo cursaron durante el año 2012-13. Y eso sin contar los alumnos matriculados en el Instituto Cervantes, o los que estudian en escuelas de idiomas privadas, datos que el Ministerio no recoge. Hay que destacar que la mayor concentración de estudiantes se encuentra en la universidad donde calculan que 22.280 alumnos estudiaron español en sus aulas en el curso 2012-13. Es decir, más de un 70\% de los estudiantes de español en China lo hicieron en el ámbito universitario en el curso 2012-13, pensando en este idioma como una posible salida profesional. No en vano, es un hecho que China cada vez tiene más relaciones comerciales con España y Sudamérica, países hispanohablantes.

Sin embargo, para un correcto uso de la lengua - especialmente tratándose de una cultura tan diferente- es preciso que los estudiantes chinos reciban una instrucción adicional en aspectos relacionados con la cultura española. Porque aunque un error gramatical, como el mal uso del subjuntivo o conjugar erróneamente un verbo irregular puede resultar hasta gracioso, un malentendido cultural podría ocasionar una actuación inadecuada o cuanto menos, incomprensible. Pensemos por ejemplo en la despedida española: "ya te llamaré". En este sentido, resulta importante que el material que se usa en el aula -entre ellos el libro de texto- haga referencia a estos contenidos culturales de forma suficiente y exacta, con el fin de que el estudiante sea capaz de expresarse con eficacia en esa cultura.

Desde los años 80 se considera que, para poder construir y descodificar significados -además de la gramática, semántica y fonética-, también es importante conocer los contenidos culturales y los valores de la sociedad a la que pertenece nuestra lengua meta. En este sentido, para un correcto uso de la lengua, es preciso ayudar al alumno a entender los distintos modos de pensar incluidos en el idioma objeto de estudio.

En esta investigación analizamos el contenido cultural del manual "Español Moderno", el libro de texto que se usa en las universidades chinas desde los años 60. Se trata de un libro muy popular, manual de referencia en la universidad, compuesto por 6 volúmenes, y editado por y para nativos chinos. Para nuestro estudio, nos vamos a centrar en los tomos uno y dos, que son los manuales que se estudian en el primer año de carrera y que podríamos decir que equivaldrían al nivel A1 del Marco Común Europeo de Referencia para las lenguas. El objetivo es comprobar, si tal y como afirma Lourdes Miquel (2004) en torno el tratamiento de la cultura en los enfoques tradicionales, existe en éstos manuales del "Español Moderno" una visión estereotipada de la cultura y una desconexión entre los objetivos de lengua y los elementos culturales, o si por el contrario, el tratamiento y enfoque sobre los contenidos culturales es el correcto. 


\section{El conocimiento cultural en el aprendizaje de una lengua}

Cuando aprendemos una lengua, el objetivo debe ser tener capacidad de comunicación pero en el contexto de la sociedad, de la cultura en la que vamos a usar esa lengua. Generalmente tendemos a seguir las pautas de conducta sociales nativas, que son las que conocemos, entendiéndolas como universales. No obstante, cuando hacemos esta interpretación en otro contexto cultural, donde las claves son diferentes, es cuando se producen los malentendidos, errores que son debidos a este desconocimiento cultural. Se da la situación entonces de que los dos hablantes -el nativo y el no nativoestán compartiendo el mismo idioma, con la misma gramática y léxico, sin embargo, cada uno está pensando en su propia lengua.

Por tanto, en el aprendizaje de un idioma no sólo es importante la competencia lingüística y gramatical sino también, en palabras de Saville Troike, ponerlo en contacto con el "conocimiento social y cultural que se les supone a los hablantes y que les permite usar e interpretar las formas lingüísticas” (Miquel, 2004, p. 141).

Imaginemos por ejemplo dos sociedades, la china y la española, donde existe un gran salto cultural. La forma china de saludarse es con la expresión: 你吃饭了吗? Ni chi fan le ma? Que en realidad significa: ¿Has comido ya?. No deja de ser un simple saludo como: "Hola, ¿qué tal?" al que se responde: "Sí, he comido" o "No, no he comido". Pero si el hablante no conoce esta fórmula cultural y codifica ese mensaje desde su lengua materna -en este caso la española- seguramente pensaría: ¿Y a esta persona que le importa si he comido o no? O igual le contestaría exactamente lo que ha comido, dónde y con quién, provocando un desconcierto en el nativo chino. Este tipo de fallos de comunicación intercultural pueden causar inseguridad por un lado y actitud negativa o rechazo por el otro.

Una artista china-alemana Yang Liu ha plasmado estas diferencias culturales en un libro, llamado "East meets west" (Taschen, 2015). Estos dibujos merecen un vistazo para la reflexión. El color azul representarían a occidente, los puntos son personas, y el rojo la representación de la comunidad china. 
Figura 1: Representación, según Yang Liu, del diferente comportamiento en las fiestas de occidentales y chinos.

\section{Durante las fiestas}

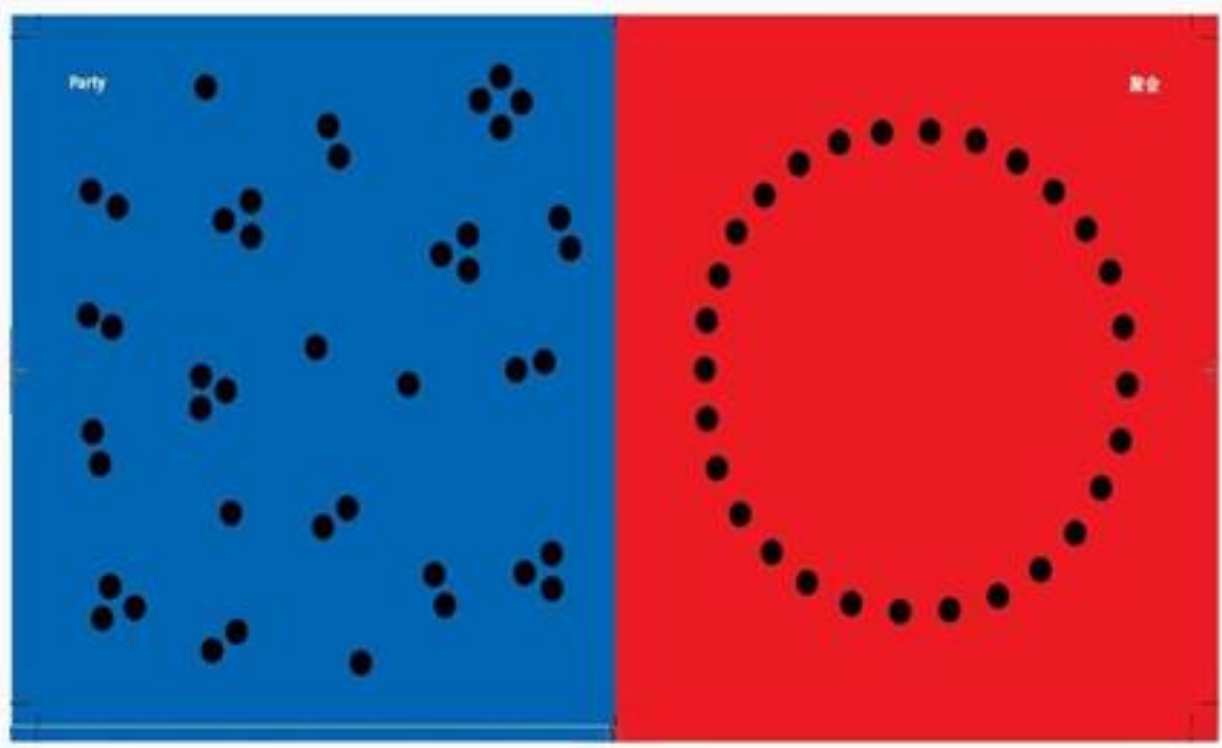

Fuente: http://bsix12.com/east-meets-west/ Pictogramas: Yang Liu.

Figura 2: expresión de la opinión, representada por Yang Liu, de occidentales y chinos.

\section{Expresión de la opinión}

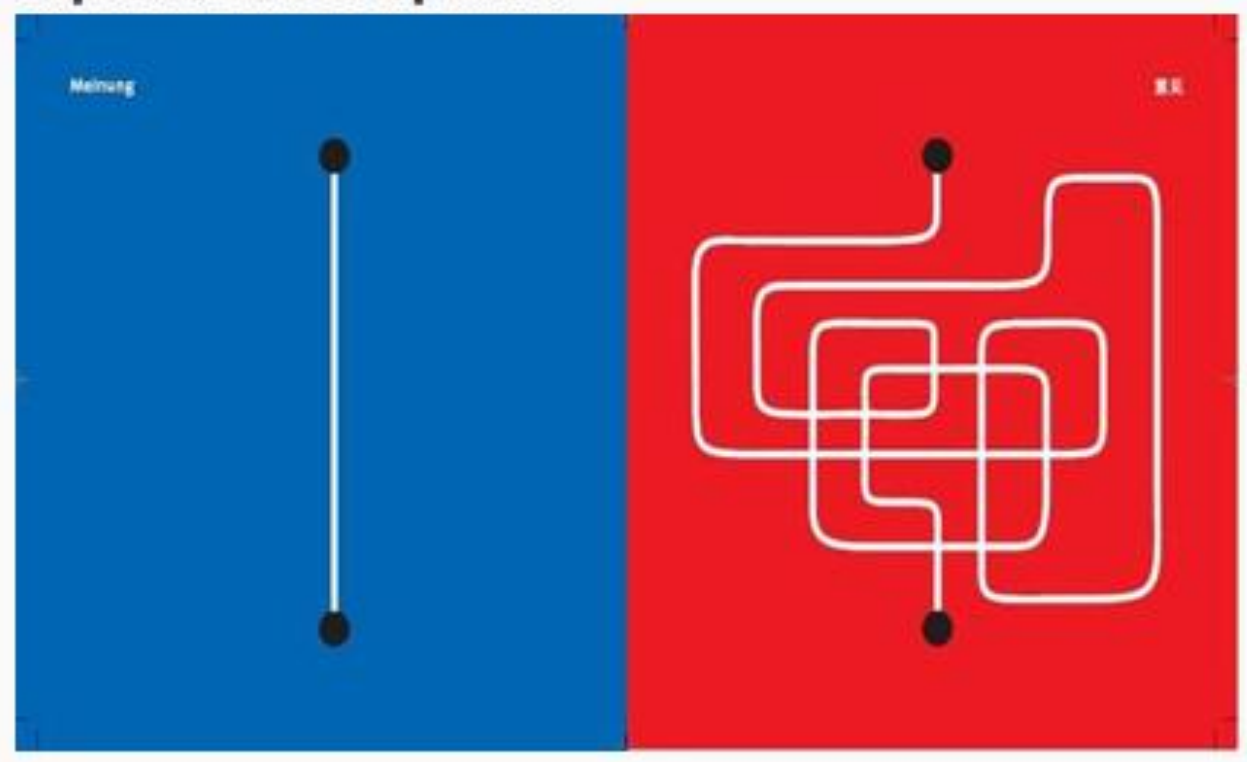

Fuente: http://bsix12.com/east-meets-west/ Pictogramas: Yang Liu.

Mirando este segundo dibujo, imaginemos la situación, en un contexto cultural español, de un chino dando su opinión sobre un asunto en un español más que aceptable. Efectivamente está comunicando y además muy correctamente, pero probablemente el oyente nativo se pondría nervioso o inquieto esperando una respuesta 
clara; y si fuera al contrario, el nativo chino pensaría que el español es demasiado directo, descarado o brusco con las opiniones, lo que crearía una situación incómoda.

Por tanto, aunque en la enseñanza de lenguas extranjeras la sintáctica y la semántica son importantes en el proceso de enseñanza-aprendizaje, no debemos olvidar el componente cultural; y es que, lengua y cultura van unidas. Porque si como afirma García (2004, p. 2), "la cultura se conforma a partir de un comportamiento aprendido y adquirido al estar el individuo inmerso en una sociedad" podemos imaginar lo que es el estudio de un idioma en un contexto de no inmersión y con unas diferencias culturales tan grandes. Obviamente hay más factores que influyen en esta adquisición de la cultura, como el método empleado por el profesor o el profesor mismo, pero resulta también muy importante el uso del manual como vehículo para comprender la cultura inherente en una comunidad.

Miquel (2004) entiende que la competencia cultural ha de abordarse contextualizada culturalmente por medio de un análisis pragmático de la lengua en el aula, presentando en el artículo bastantes ejemplos cotidianos que en realidad son actos de cultura, como rechazar o aceptar un ofrecimiento, reaccionar cuando recibes un regalo, presentar a una persona etc. Por tanto, es necesario abordar la cultura desde su uso, analizando situaciones concretas para darle significado, contextualizando las muestras de cultura.

De este modo, parece claro que en el aprendizaje- enseñanza de una lengua se debe incluir también la cultura. De hecho "es imposible enseñar una lengua sin hacer constante referencia a la cultura de sus hablantes, ya que la lengua se refiere implícitamente a la percepción del mundo y a los códigos de conducta de aquellos" (Soler-Espiauba, 2009, p.219).

Pero ¿qué se entiende por cultura? Se trata de un concepto abordado desde diferentes perspectivas y disciplinas y sobre el que se han propuesto numerosas definiciones. Según la definición de la Real Academia española de la lengua en su acepción número tres, cultura es un conjunto de modos de vida y costumbres, conocimientos y grado de desarrollo artístico, científico, industrial, en una época, grupo social, etc. Las autoras Miquel y Sans (2004) propusieron la ya famosa división entre Cultura con mayúsculas, cultura a secas y cultura con k. La Cultura con mayúscula se refiere a toda la cultura enciclopédica, y la cultura con $\mathrm{k}$ a una cultura más underground. Es en la cultura a secas donde las autoras consideran que deberían caer los esfuerzos didácticos por ser esta más dinámica y porque es aquí donde se encuentran todos lo estándares culturales compartidos que permitirían al estudiante entender la realidad cotidiana y comportarse e interactuar correctamente. $\mathrm{Y}$ es en ésta última donde nosotros también vamos a fijarnos. Este tipo de cultura también es denominada por Navarro (2009) como cultura epidérmica, puesto que la sitúa más en el terreno de lo superfluo e inconsciente; esta cultura está dotada de elementos que normalmente pasan desapercibidos a los hablantes nativos de una lengua. 
Otra buena definición de este tipo de cultura sería: "Todo lo compartido por los ciudadanos que conviven en un determinado ámbito, y que se da por sobreentendido". (Soler-Espiauba, 2009 , p. 218). Autores como Porcher (Zhu, 2010, p. 24) hablan de cultura como una "ficha de identidad de una sociedad [...] fundada más en convicciones que en un saber". O Harris (Zhu, 2010, p. 24) que ve la cultura como un "conjunto aprendido/adquirido socialmente de tradiciones, estilos de vida y modos pautados y repetitivos de pensar, sentir y actuar". Miquel y Sans (2004) hacen una importante apreciación, y es que el estudiante no solo ha de tener conocimientos "sobre" la cultura, sino que estos conocimientos le tienen que servir para actuar en la sociedad o con individuos que hacen uso de la lengua-meta. Es por ello que los autores abogan por el uso de la competencia comunicativa como acercamiento a la competencia cultural.

\section{Aprendizaje del español en China}

En primaria y secundaria la metodología de enseñanza de idiomas en China sigue siendo el método tradicional basado en gramática-traducción. Dentro del ámbito universitario, sin embargo, el método tradicional de enseñanza parece que está empezando a cambiar en beneficio de los enfoques por tareas o con la implantación de un método más comunicativo (Yang, 2015). No obstante, ¿cuál es la razón por la que siguen usando el mismo libro en las universidades hasta el día de hoy? Los artículos de Mosquera (2011 ) y Sánchez (2009) atienden a dos motivos: Por una lado, la tradición confuciana de los exámenes imperiales basados en la memorización de textos y en las reflexiones a partir de unas explicaciones concretas. Sirva como ejemplo que las doce primeras lecciones del Tomo I, tienen un ejercicio al final de la lección que literalmente dice: "Lee el texto y aprende de memoria la primera parte". Otra razón de su perseverancia, es la configuración del sistema de exámenes nacionales o los exámenes para la obtención de diplomas o para ingresar en un postgrado, bien expedidos por universidades chinas o bien por instituciones extranjeras. En todos ellos pesan mucho más las destrezas escritas y la adquisición de vocabulario que las destrezas orales o lo que hoy llamamos "competencia comunicativa". Valga como ejemplo la lección 8, pág. 119, del tomo I del manual "Español moderno"; A continuación, presentamos el texto que explica cómo los alumnos chinos estudian lengua extranjera. “¿Cómo la aprendemos? Primero la profesora lee el texto y explica las palabras nuevas. Después hace preguntas y nosotros tenemos que contestar. También tenemos que comentar en texto. Podemos comentarlo en chino o en español".

Si como venimos diciendo, es importante que el contexto sociocultural esté presente en el aula, no es menos importante que esta visión cultural no sea una visión simplista y alejada de la realidad. A veces, las visiones distorsionadas de la cultura se sustentan en estereotipos, definido por el Diccionario de la Real Academia Española en su primera acepción como una "imagen o idea aceptada comúnmente por un grupo o sociedad de carácter inmutable" (Real Academia Española , 2014). Para Lamo de 
Espinosa (1993, p.13) "un estereotipo sería la simplificación de la realidad poco conocida para hacerla manejable y comprensible". El Plan Curricular del Instituto Cervantes considera los estereotipos como señas de identidad de la propia cultura. Hay que tener especial cuidado con los estereotipos e intentar que haya un tratamiento adecuado de ellos en los manuales porque, para muchos estudiantes, este será el primer contacto y seguramente el único con la cultura meta. Tal y como afirma Kramsch (1993), muchas veces nos quedamos solo en lo superficial, y nos basamos en la descripción de la cultura en referencia solo a las cuatro "efes": facts, food, folklore y festivals. Esto es: festividades típicas, los bailes, el folklore y la comida. En concreto, España tiene un pesado lastre en estereotipos forjado primero en los años 50 con el famoso Spain is Different, que dio una visión exótica y folclórica de nuestro país, y después por las campañas publicitarias turísticas de los años 50, 60 y 70 que difundieron la imagen de una España soleada y costera. Es cierto que los estereotipos sobre España están cambiando y se están forjando nuevos como por ejemplo el fútbol, pero verdaderamente son tan fuertes que aún perduran. Por ejemplo, en el boletín económico sobre la imagen de España en China del 2009 se afirma que "9 de cada 10 chinos que conocen España nombran de forma espontánea la tauromaquia, mientras que el 53 por 100 nombran el fútbol” (Laso \& Justo, 2009 , p. 98) .

\section{Planteamiento de los contenidos culturales según el Plan Curricular del Instituto Cervantes}

El Instituto Cervantes (IC) ha realizado el difícil trabajo de desarrollar y analizar la dimensión cultural desde un punto de vista comunicativo y no tanto lingüístico que ha recogido en tres inventarios: los Referentes Culturales, los Saberes y Comportamientos Socioculturales y las Habilidades y Actitudes Interculturales. En éstos, resume un tipo de conocimiento que llama "factual", la Cultura con mayúscula de la que hablábamos anteriormente, junto con: creencias, valores, representaciones y símbolos. En concreto, el inventario Saberes y Comportamientos Socioculturales, que es el que nosotros tomaremos como referencia para nuestro análisis, está estrechamente relacionado con los Referentes culturales, tal y como este apartado recoge en su introducción en cuanto a las "condiciones de vida, convenciones sociales, comportamientos rituales, valores y creencias respecto a las realidades, y aspectos sociales y culturales" (Instituto Cervantes, 2006, párr. 13). Todas las especificaciones de este apartado se refieren sólo a España haciendo alusión "al conocimiento, basado en la experiencia, sobre el modo de vida, los aspectos cotidianos, la organización social, las relaciones personales, etc." (Instituto Cervantes, 2006, párr.2) que se dan en nuestro país. Suponemos que el no haber realizado un inventario específico sobre cada país hispano se debe al elevado volumen de países que hablan español, de hecho, veinte lo tienen como lengua oficial o cooficial. Efectivamente, según apunta el Plan Curricular, los contenidos culturales que recoge son relativos sobre todo a España por la "dificultad que conlleva realizar un análisis de la enorme variedad de aspectos que se dan en cada una de las sociedades de 
los países hispanos y ofrecer una presentación adecuada" (Instituto Cervantes, 2006, párr. 2). Estos saberes culturales llevan asociados una serie de creencias y valores que se manifiestan en los miembros de la sociedad y que están relacionados con las ideas, los prejuicios, las convicciones, los estereotipos, etc. Por otro lado, los comportamientos culturales están relacionados con las convenciones sociales y se refieren a las situaciones de interacción (Instituto Cervantes, 2006 párr. 3, 4). El inventario además tiene tres fases: aproximación, profundización y consolidación. Estas no tienen relación directa con los niveles del Marco Común Europeo para las lenguas: A, B y C . Los criterios que han seguido para establecer la distribución en las tres fases son la universalidad y la accesibilidad, que resulten más o menos necesarios para los contactos y las interacciones de los alumnos. Así, en el nivel de aproximación se presentan los contenidos relacionados con temas más cercanos a sus experiencias cotidianas (Instituto Cervantes, 2006 párr. 7).

\section{Método}

Nos disponemos a realizar un análisis de contenido cultural del manual de español "Español Moderno", libro de referencia que se utiliza en las universidades chinas donde se encuentra el grueso de los estudiantes de español, más de un $70 \%$ de ellos lo estudiaron en la universidad durante el curso 2012-13. Este manual es el más utilizado en la asignatura principal, lectura intensiva, de la carrera de Filología Hispánica en China (Yang, 2015). Para realizar el análisis de los contenidos culturales que se abordan en el manual, utilizamos aquellos contenidos culturales ya recogidos en el Plan Curricular del Instituto Cervantes, en concreto, los que aparecen en el apartado Saberes y Comportamientos Socioculturales en su fase de aproximación. Además, hacemos un análisis de los estereotipos sobre la cultura española presentes en el manual.

\section{Descripción de la muestra}

El manual de español "Español Moderno" está escrito por hispanistas chinos para un estudiante adulto cuya lengua materna es la china. La editorial y la impresión también pertenecen a este país. El mismo autor que escribió el manual Español Moderno en los años sesenta, Dong Yangsheng, es el que se ha encargado de hacer las ediciones posteriores y las modificaciones de las mismas. Dong Yangsheng es reconocido por ser el más célebre traductor de Cervantes al chino.

El "Español Moderno" se compone de seis tomos. La primera edición de los dos primeros se publicó en 1999 y los siguientes en los años 2000, 2001, 2003 y 2007 respectivamente. La muestra que vamos analizar pertenece a los manuales I y II, los que se estudian en el primer año de carrera y que podríamos situar, por tener una referencia aproximada, dentro del nivel A del Marco de Referencia Europeo. La edición que hemos cogido es la última, la del 2007. El manual I tiene veinticuatro lecciones y el 
manual II dieciséis lecciones. Por tanto, en total, nuestras unidades de análisis son estas cuarenta lecciones de las que constan los dos manuales juntos.

La estructura de cada lección es fija y ordenada. Presenta primero un pequeño texto narrativo, complementado a veces con un segundo en forma de breve diálogo. Seguidamente y por este orden, aparece el vocabulario, la parte gramatical y los ejercicios que por regla general son de traducción directa e inversa y de rellenar huecos. Las muestras de lengua analizadas pertenecen a los textos de cada lección, puesto que es ahí donde está presente el contenido cultural. De esta manera, los textos de cada lección son nuestras categorías de análisis.

\section{Recogida y análisis de datos}

Para codificar los contenidos sobre la cultura española presentes en el manual tomamos como referencia el punto 11 del Plan Curricular del Instituto Cervantes: Saberes y Comportamientos Socioculturales . A su vez, este apartado está dividido en tres categorías representadas en las Figuras 1, 2 y 3: Condiciones de Vida y Organización Social; Relaciones Interpersonales e Identidad Colectiva; y Estilo de Vida.

Cada una de estas tres grandes divisiones a su vez, está subdividida en otros apartados que contienen descripciones menores y que, en la mayoría de los casos, vuelven a desarrollarse en instancias más pequeñas. Por ejemplo: Comidas y Bebidas, que se enmarca dentro de Condiciones de Vida y Organización Social, tiene tres subdivisiones: Cocina y Alimentos; Convenciones Sociales y Comportamientos en la Mesa; y Establecimientos; y, cada una de ellas, contiene ocho, tres y cuatro subdescriptores respectivamente. En total, hemos contabilizado 149 contenidos culturales repartidos en cada una de las categorías principales: Condiciones de Vida y Organización Social (121); Relaciones Interpersonales (13); e Identidad colectiva y Estilo de Vida (15). 
Figura 3. Condiciones de vida y Organización Social: apartados y número de subapartados contemplados en el Plan Curricular.

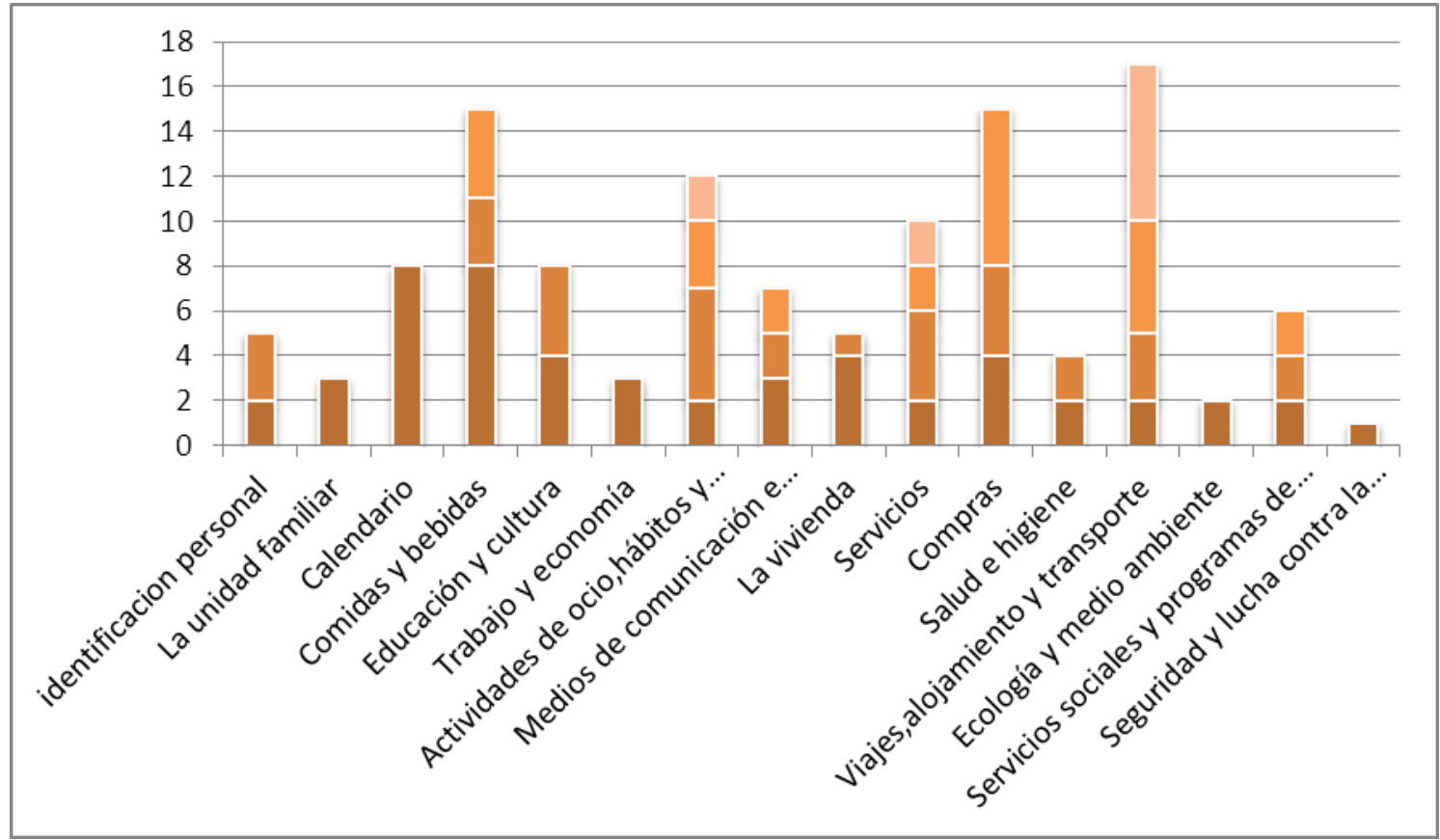

Fuente: Gráfico de elaboración propia

Figura 4. Relaciones Interpersonales: apartados y número de subapartados contemplados en el Plan Curricular.

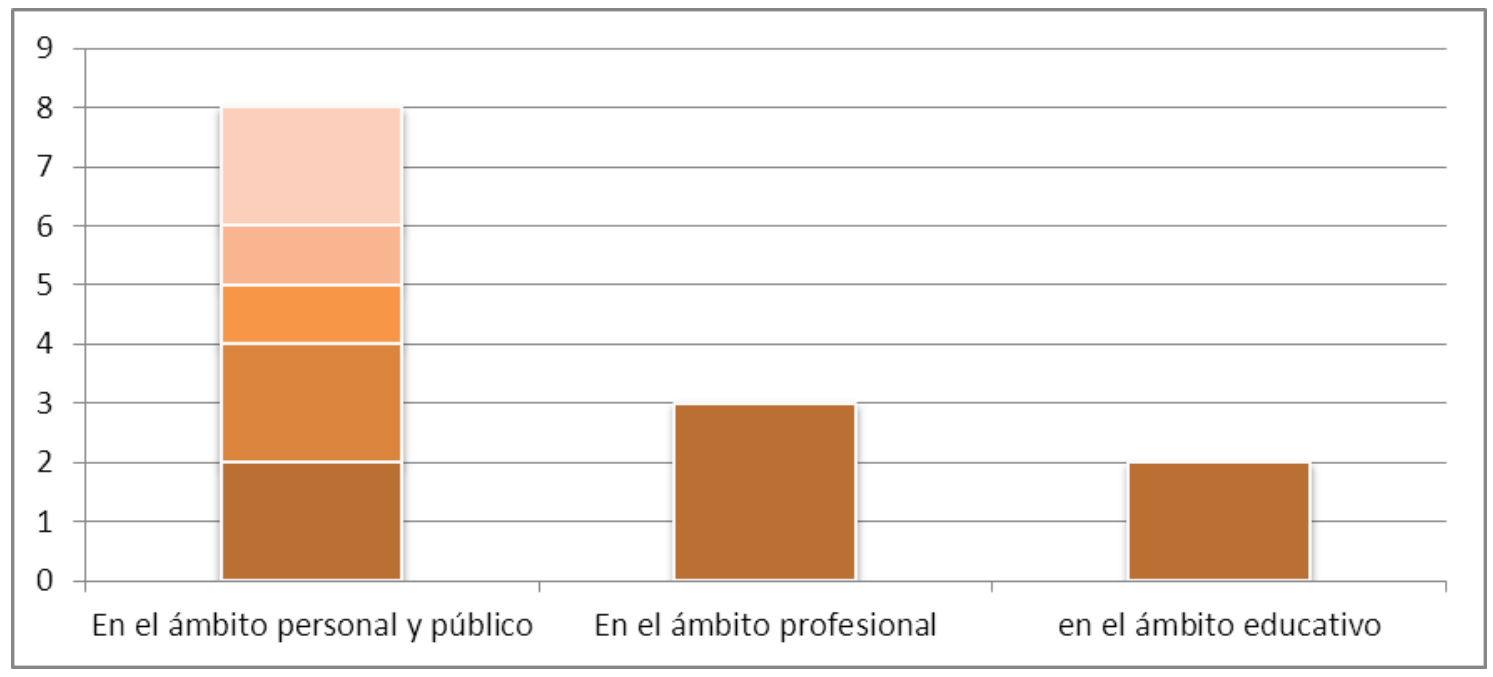

Fuente: Gráfico de elaboración propia 
Figura 5. Identidad Colectiva y Estilo de vida: apartados y número de subapartados contemplados en el Plan Curricular.

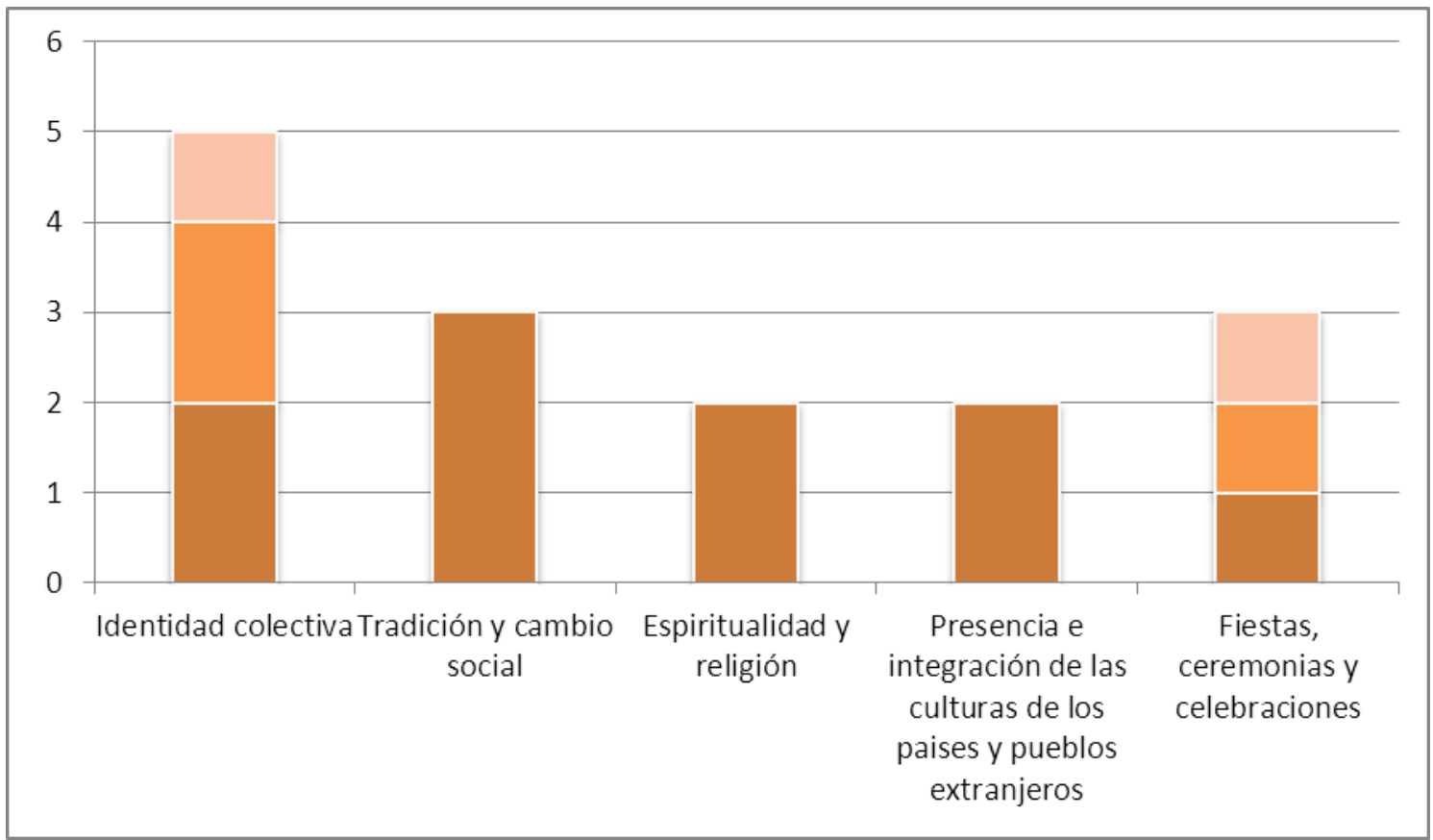

Fuente: Gráfico de elaboración Propia

Tomando como referencia estas categorías recogidas en el Plan Curricular del Instituto Cervantes, pasamos a cuantificar aquellas presentes y ausentes en el Manual Español Moderno.

\section{Resultados}

Analizando los dos tomos del manual Español Moderno se han encontrado 24 apariciones de referentes culturales que podrían ser españoles de los 149 que presenta el Plan Curricular, esto es un $15,4 \%$. 
Figura 6. Resultados totales. Comparativa del número de contenidos culturales que plantea el Plan Curricular y los que aparecen en los tomos I y II del manual "Español Moderno".

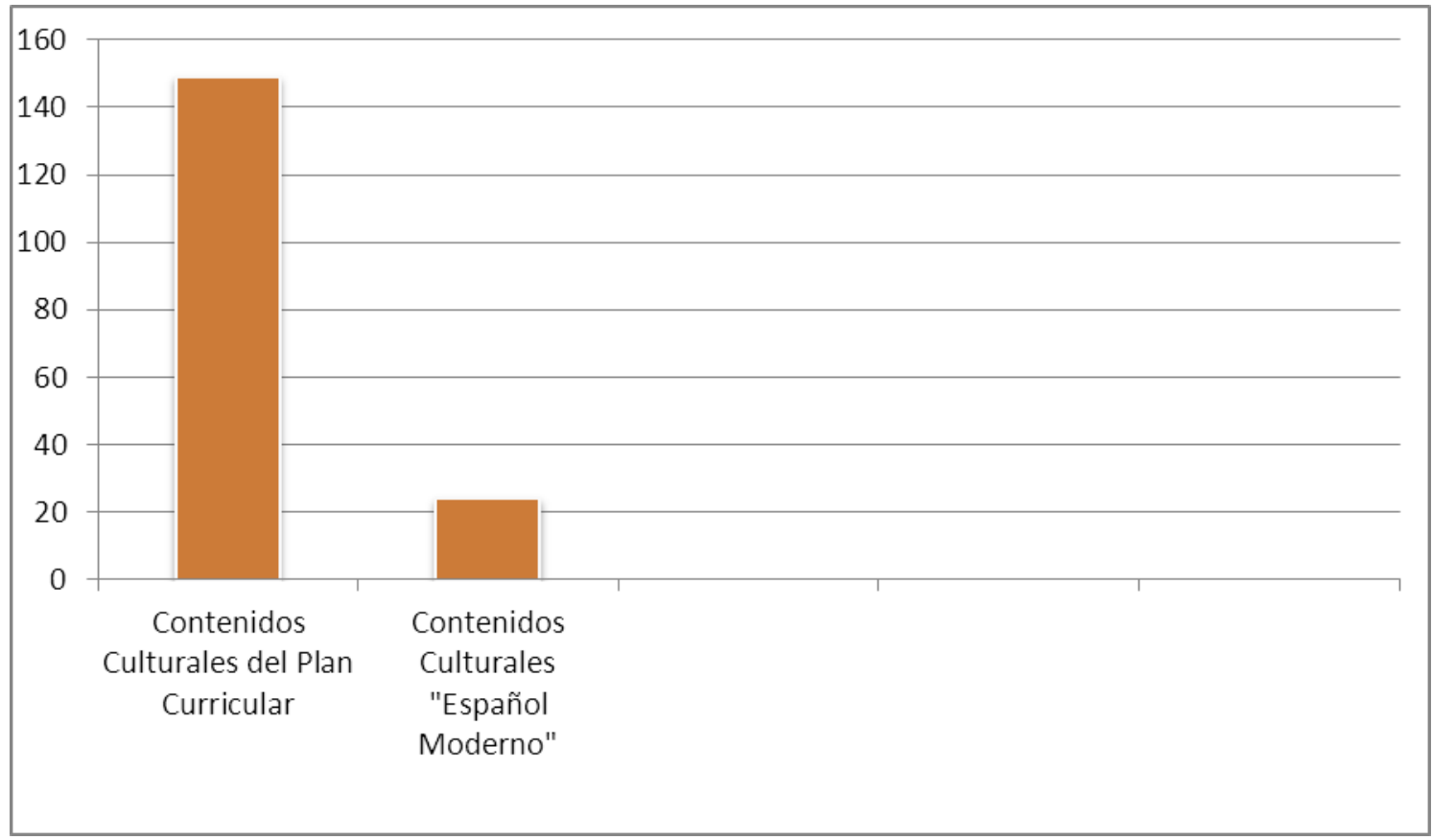

Fuente: Gráfico de elaboración propia

La mayor parte de los referentes culturales se encuentran dentro del primer gran bloque: Condiciones de Vida y Organización Social, ya que contiene 21 de los 24 resultados totales obtenidos, un dato que no es muy notable si tenemos en cuenta que este bloque consta de 121 descriptores como muestra la Figura 1.

Hay que precisar que la mayoría de las veces estos referentes culturales que hemos contabilizado están tratados vagamente y son un tanto imprecisos en relación con la descripción más explícita que suele aparecer en el inventario. Pondremos algunos ejemplos: en el apartado de Comidas y Bebidas, que tiene un total de 8 subcategorías, hemos contabilizado 3. Sin embargo, en este apartado se lee: "Horarios de las comidas principales y alimentos asociados a cada comida. Conceptos de tapa y merienda". Hemos contabilizado como presente, cuando en realidad solo aparece la primera parte, es decir, los horarios de comidas. No hay ninguna referencia a los alimentos respecto a éstas, y tampoco a los conceptos de tapa y merienda. Con el apartado de Trabajo y Economía ocurre algo similar. Encontramos y contabilizamos 2 de las 3 categorías que aparecen en el inventario, a pesar de que en el inventario se recoge "Horarios de trabajo, descansos y vacaciones". En nuestro análisis lo contabilizamos como presente ya que en el manual se habla de que los domingos no se trabaja, aunque no se hace referencia a vacaciones ni a jornadas de trabajo.

El apartado de Compras, que tiene en total 15 descriptores, solamente aparece 1 que corresponde a la interacción cliente-dependiente, dejando sin tratar los otros 14 descriptores, como por ejemplo: el tipo de establecimientos, concepto de rebajas, 
convenciones en la forma de colocar los precios 9,99€, incluso las formas de pago, que en los textos no aparece y sin embargo sí que lo hace la moneda local, esto es, el yuan.

Igualmente la parte de Viajes, Alojamiento y Transporte no se trata en profundidad, apareciendo un total de 2 descriptores, de los 17 recogidos. Además, existen seis categorías adicionales que no se ven representadas por ningún descriptor (Figura 5).

Figura 7. Resultados obtenidos en el apartado Condiciones de vida y organización social, donde se encuentran la mayoría de los referentes culturales.

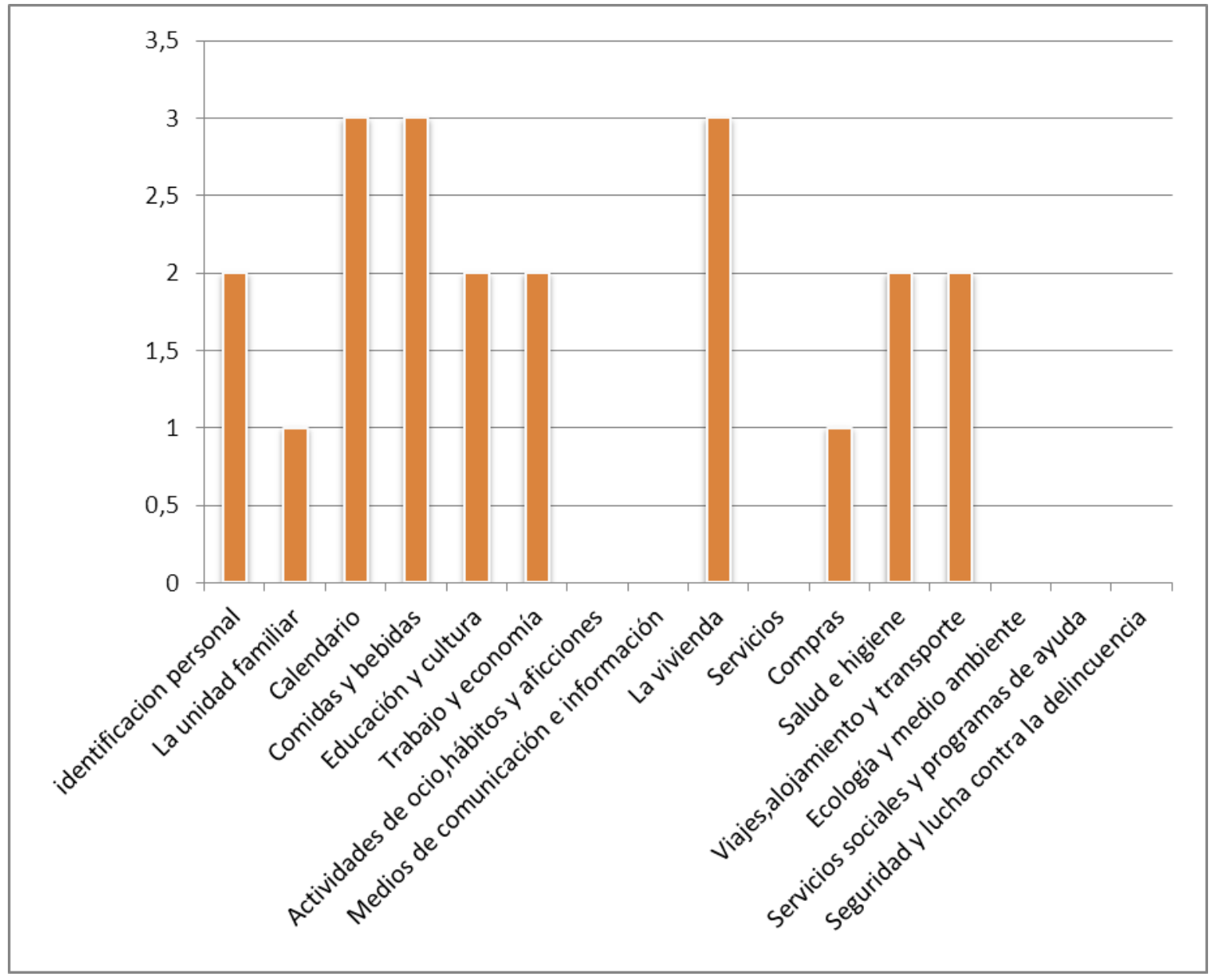

Fuente: Gráfico de elaboración propia

\section{Errores y descontextualizaciones}

Otro de los grandes apartados, el de Relaciones Interpersonales (Figura 2), solo incluye 1 de los 13 marcadores que el Plan Curricular propone, siendo éste el del ámbito personal y público, y las relaciones sentimentales, familiares y de amistad, que aparecen varias veces de forma explícita en la lección 17 del tomo I, aunque está tratado de forma poco natural y un tanto forzada: 
- ¿Qué prefieren beber?

- Sírvannos té de jazmín. [...]

- Ahora mismo se lo traigo. Pero tienen que perdonarme, porque luego tengo que meterme en la cocina y no voy a poder acompañarlos.

Otro ejemplo de estas inexactitudes se encuentra en la lección 21, también del tomo I. Se trata de un texto relativo a una pareja que llega tarde a la reunión con amigos. En el texto se lee: Cuando se saludaban y abrazaban, se excusaron del retraso.

En cuanto al último gran apartado (Figura 3), Identidad Colectiva y Estilo de Vida, que cuenta con 15 categorías -entre ellas las fiestas populares, las creencias religiosas, las celebraciones y convenciones en los cumpleaños, etc.-solamente hemos encontrado 2 en los dos tomos del manual, siendo éstos solamente enunciados, sin ninguna otra referencia. Se trata de la Navidad y del hecho de que los regalos se abren cuando se reciben.

Por otro lado, en nuestra contabilización total habíamos dicho que estos referentes "podrían" ser españoles porque nos ocurren dos cosas, o bien los textos donde se presentan están descontextualizados -y no sabemos si los personajes están en España o en China-, o bien el contexto, implícito o explícitamente, es totalmente chino. Por ejemplo, esto se aprecia cuando se abordan contenidos relacionados con la visita al hospital al que acuden los personajes por un resfriado. No es un contenido del todo exacto pues en España iríamos al ambulatorio o al centro de salud, términos que aparecen especificados en nuestro inventario. No obstante en China la traducción literal para indicar que vas al médico es utilizar la palabra hospital. Igualmente los síntomas que muestra el enfermo, son diálogos que culturalmente nosotros no utilizaríamos, aunque en la cultura China sería normal preguntar y hablar sobre este tipo de cuestiones. Véase un ejemplo:

- Tenía una indigestión.

- Es una enfermedad muy molesta. Sentías hinchado el estómago, ¿verdad?

- ¡Cómo no! Además eructaba mucho. Y mi aliento era muy feo.

Existen más ejemplos de textos descontextualizados, cuyos personajes parecen, de hecho, localizarse más en China que en España. Como ejemplo tenemos la lección 16: De Compras. Se podría pensar que el personaje se encuentra en un contexto español o hispano, no obstante, un diálogo -parte del cual reproducimos a continuación- revela que el cliente paga en yuanes.

- Espere, veo por allá unas camisas. Parecen de buena calidad. Haga el favor de sacármelas.

- Aquí las tiene. De veras son muy buenas, pero son de diferentes precios. Por ejemplo, las azules son más caras que las blancas, y estas más caras que las amarillas. Las de color café son tan baratas como las rojas. (...) 
- Me queda bien, ¿verdad?. Bueno me las llevo ¿Cuánto le debo?

- Son 186 yuanes.

Hay otras veces que en un contexto chino se presentan ciertas costumbres españolas, como a la hora de tratar a las visitas con motivo fiesta nacional china del $1^{\circ}$ de octubre, ocasión que se podía haber utilizado para presentar una fiesta española como contenido cultural, tal y como recomienda el Plan Curricular. La parte de visitas turísticas también se contextualiza completamente en Pekín, así como el tema de Prestaciones Sociales, que da título a la lección 14 del tomo dos.

Pero dentro de esta escasez, hallamos tres descriptores que son más o menos correctos y que también merecen atención. Uno son los textos pertenecientes a las lecciones 22 y 23, los únicos ubicados en España, concretamente en Madrid, donde un joven alumno chino ha ido a estudiar. Es en estos textos donde se encuentra el grueso de la representación de la cultura española que hemos encontrado en los manuales. En ellos se habla del metro, de lo caro que son los alquileres en Madrid y de que con el carnet de estudiante se entra gratis al Museo del Prado. A pesar de todo, no nos dejan de sorprender las analogías con China. Por ejemplo el protagonista, que es consciente de que vivir en un Colegio Mayor es más caro que en un piso, se decanta por vivir allí, igual que ocurre en China, donde la inmensa mayoría de los estudiantes viven en los campus universitarios.

Los otros descriptores culturales que también están tratados más consistentemente son los que tienen relación con la rutina diaria, las comidas y sus horarios. Los dos temas están expuestos realizando una comparación con la cultura propia. Por ejemplo, el tema de la comida, cuyas costumbres se contrastan con las de Occidente, contiene algunas inexactitudes como vemos en el siguiente ejemplo de la lección 15 del Tomo I pág. 228 del "Español Moderno":

"Es notable la diferencia. Aquí los platos pueden ser muchos o pocos, pero siempre se sirven al mismo tiempo en la mesa y se comen con arroz o pan. Los chinos comemos con palillos. En Occidente, los platos no son muchos. La comida comienza con un plato frío, luego viene la sopa. El primer y el segundo plato se sirven uno tras otro. Por ultimo, el "postre" y el café".

También hemos encontrado errores en la forma de hablar, no gramaticalmente que son correctas, pero si en el uso, y es que las muestras de lengua son un tanto irreales. En concreto, de los 37 diálogos que se incluyen en los dos tomos, solo en 3 de ellos la muestra es correcta y natural, tal y como un nativo utilizaría el idioma. Es decir sólo un $8,1 \%$ de las muestras de lengua de los diálogos son exactas y reales.

A continuación mostramos algunos ejemplos más de muestras de lengua inexactas desde el punto de vista comunicativo:

Lección 15 pág. 229, tomo I "La comida"

- A ver, ¿Qué deseas comer? 
- De primero una ensalada ¿Qué dices?

- Está bien, si es de verduras me gusta más.

Lección 17 pág. 265, tomo I "Una visita”

- De acuerdo. Entonces la botella está ahí y servíos vosotros mismos. Ya sabéis: estáis en vuestra propia casa. Ahora decidme: ¿Qué queréis de mí?

Lección 4 pág. 73 Tomo II "Primera vez como interprete"

- No se precipite demasiado. El tráfico está muy peligroso.

Lección 21 pág. 342 Tomo I "El tráfico de la ciudad"

- Pero cuando sacaron a todos los heridos y muertos, ¿No pudisteis volver a conducir?

\section{Estereotipos}

En relación con los estereotipos no hemos observado muchos ejemplos, lo cual es comprensible porque, como venimos diciendo, el libro se encuentra más bien en un contexto chino. Para el estudio escogimos siete estereotipos que Hernández Cortés (2014) enumera en su trabajo como universales, y además añadimos la variable "fútbol" que el barómetro del Real Instituto Elcano apunta en su último estudio hecho en 2015. En definitiva estos son: toros, flamenco, siesta, fiesta, buen tiempo/sol y playa, fútbol, "los españoles son todos morenos", "los españoles son simpáticos y alegres y también poco trabajadores".

Tras nuestro análisis, encontrado solamente un estereotipo español, el flamenco, y lo hemos hecho en dos ocasiones. Contabilizaríamos una tercera si tenemos en cuenta que la portada del libro es una silueta de una bailaora de flamenco con un torero dando un muletazo a un toro. No obstante, el estereotipo del flamenco está tratado de forma aceptable, ya que la situación que se presenta es la de dos personajes que piden a un español que les enseñen a bailar flamenco. El español les contesta que él tampoco sabe, que es muy difícil; quizás no sea tan realista que el personaje resuelva discretamente la situación diciéndoles que les enseñará a bailar sevillanas. También la fiesta de Nochevieja, la única fiesta española de la que se habla en el manual, y que el Plan también incluye en su inventario, aparece una vez en cada tomo.

Por otra parte, en la lección 2 del tomo II, donde se hace una representación del folklore hispánico, incluido el flamenco y las sevillanas el texto apunta: "tuvimos la oportunidad de conocer un poco la cultura hispánica". De hecho, se hace uso en varias ocasiones de esta expresión "cultura hispánica", como si fuera un contenido importante a tener en cuenta, aunque como vemos el libro no lo trata apropiadamente.

Como curiosidad, encontramos en el manual una referencia a un choque cultural que sufre frecuentemente la comunidad china cuando se relaciona con la española. Se 
trata del agradecimiento con el que los occidentales suelen responder a los halagos. Esta costumbre resulta chocante para los asiáticos, ya que en su cultura lo educado es negar humildemente los cumplidos y restarles importancia. Pero en España, como bien dice Soler-Espiauba (2009), si no agradecemos a quien nos alaba contribuimos a desvalorizarle, y el manual así lo contempla.

- ¿Qué frío! Bonita casa tiene usted.

- ¿Verdad?, Gracias.

Observamos, sin embargo, un pequeño error en el tomo dos de la lección 3, titulado: No lo haga. En él se presentan ejemplos de preguntas que no se deben hacer en España para introducir el uso del imperativo negativo. Entre ellos, se pone como ejemplo preguntar cuánto ganas a un desconocido, algo que los chinos suelen hacer con toda naturalidad, o preguntar a una mujer su estado civil o la edad si es mayor de 25 años. Efectivamente preguntar a una persona la edad, fuera de contexto, puede resultar poco respetuoso en la cultura española.

\section{Discusión y conclusiones}

Somos conscientes de que es imposible que en los manuales de un idioma estén incluidas todas las referencias culturales de un país, especialmente del español, que es lengua oficial u cooficial en veinte países. A pesar de todo, en el estudio que hemos realizado de los tomos I y II del manual "Español Moderno" para la enseñanza del español en contextos universitarios en China, se muestra un escaso tratamiento de contenidos relacionados con la cultura española. En concreto, los resultados demuestran que aparece solamente un $15.4 \%$ del contenido cultural que debería ser enseñado según el Plan Curricular del Instituto Cervantes en su inventario Saberes y Comportamientos Socioculturales.

De las cuarenta lecciones que incluyen estos dos primeros tomos, podemos afirmar que, solamente una lección, está en un contexto cultural español y es en el único sitio donde aparece la Cultura con mayúscula. Todas las demás lecciones se encuentran descontextualizadas o se presentan en la realidad China.

Como ya apuntamos, este manual está escrito por y para chinos y que es el mismo desde los años 60, aunque con algunas variaciones en el léxico, y esa puede ser la causa de la descontextualización. En aquellos años los chinos no viajaban al extranjero y por tanto la necesidad del idioma podía ser otra. Esto lo vemos en textos relacionados con los viajes en los que son los extranjeros los que van a Pekín, y el hablante chino es el guía, u otras posibilidades de trabajo que aparecen explícitamente en otro capítulo del libro en campos como el comercio exterior, o profesiones como la de diplomático, profesor o traductor.

La presentación del contenido en el libro está al servicio de la parte gramatical. Los enunciados de las lecciones, aunque parece que están estructurados para ofrecernos 
contenido cultural, en realidad parecen más una excusa para tratar la gramática. De hecho, en lecciones en los que hay dos textos, uno narrativo y otro en forma de diálogo, a veces la única conexión que hay entre ellos es gramatical, echando de menos un hilo conductor que englobe la lección. Por ello el contenido de los textos, que además suelen ser muy breves, aparecen forzados como muestra gramatical y su explotación didáctica está dirigida exclusivamente al desarrollo de ésta.

Sin embargo, analizando el manual y sobre todo la estructura, si nos fijamos en los títulos de los capítulos, parece que los temas no están escogidos de manera aleatoria, de hecho prácticamente todos aparecen en nuestro inventario aunque como pretexto para los contenidos teóricos. A pesar de todo, se recogen algunos contenidos culturales básicos y éstos están contemplados correctamente, como la presentación de horarios en relación con las comidas o la rutina diaria. Pero, incluso en estos casos, el manual no se desliga de la cultura china, ya que el contenido aparece implícitamente explicado por medio de la comparación con ésta. Por tanto podemos afirmar, tal y como nos planteábamos en base a las afirmaciones de Miquel (2004), que los objetivos de la lengua y los elementos culturales se encuentran desconectados.

También conviene señalar la existencia de numeroso vocabulario proveniente de Hispanoamérica, lo cual seguramente esté relacionado con el hecho de que la profesora peruana Georgina Cabrera haya participado, tal y como indica el prólogo, en la elaboración de estos dos manuales. Por esta misma razón y porque, como hemos visto, el libro no está en un contexto cultural español, han aparecido tan pocos estereotipos a excepción de la portada con el toro y el torero y la alusión al baile típico español: el flamenco.

Por último, con relación a la metodología y el impacto de la dimensión cultural en el enfoque comunicativo, el hecho de que este manual se centre en el estudio de la gramática y se incluyan pocos contenidos culturales, nos hace pensar que en realidad el componente cultural y comunicativo no es tomado muy en consideración. Parece que en el estudio del idioma en la universidad primará la capacidad de aprobar un exámen, basado en gramática y traducción, por encima de la capacidad de comunicación en un contexto real. Tanto es así, que, tal y como está presentada la primera lección del manual, en el primer día de clase de español el estudiante podrá presentar a alguien y hablar en tercera persona, pero será incapaz de presentarse y hablar de sí mismo porque no se enseña la forma del verbo ser en primera persona: "soy". 


\section{Referências}

Atienza, J. L., \& Blanco, M. (2005). ¿Cómo se ven? ¿Cómo nos ven? Atrapados en los estereotipos. Oviedo: Ediciones de la Universidad de Oviedo.

Byram, M., \& Fleming, M. (2001). Perspectivas interculturales en el aprendizaje de idiomas. Madrid: Cambridge University Press.

Consejo de Europa (2002). Marco común europeo de referencia para el aprendizaje, enseñanza, evaluación. Madrid: Secretaría General Técnica del MEC, Anaya e Instituto Cervantes.

Fachal, M. (2012). ¿Por qué Beiwai es la cuna de alguno de los mejores hispanistas chinos? Tinta China , 7, 8-10.

Fachal, M. (2012). Entrevista a Dong Yangsheng . Tinta China , 7, 11-12 .

García, P. (2004). La cultura, ¿universo compartido? La didáctica intercultural en la enseñanza de idiomas. RedELE, 0, Ed. Ministerio de Educación y Ciencia.

Instituto Cervantes (s.f). Centro Virtual Cervantes. Referentes Culturales. Introducción . Recuperado de http://webcache.googleusercontent.com/search?q=cache:1Yk4cazVFIAJ:cvc.cer vantes.es/ensenanza/biblioteca_ele/plan_curricular/niveles/10_referentes_cultura les_introduccion.htm $+\& \mathrm{~cd}=1 \& \mathrm{hl}=\mathrm{es} \& \mathrm{ct}=\mathrm{clnk} \& \mathrm{gl}=\mathrm{us}$

Instituto Cervantes. (s.f). Centro Virtual Cervantes. Habilidades y actitudes interculturales. Recuperado de http://cvc.cervantes.es/ensenanza/biblioteca_ele/plan_curricular/niveles/12_habil idades_y_actitudes_introduccion.htm

Instituto Cervantes. (s.f). Centro Virtual Cervantes. Saberes y comportamientos socioculturales. Recuperado de http://cvc.cervantes.es/ensenanza/biblioteca_ele/plan_curricular/niveles/11_sabe res_y_comportamientos_inventario.htm

Hernández, C. (2014). Tópicos y estereotipos españoles del siglo XXI en el aula de ELE. Universidad de Oviedo: Recuperado de http://hdl.handle.net/10651/27990

Kramsch, C. (1993). Context and Culture in Language Teaching. Oxford University Press.

Lamo de Espinosa, E. (1993). La mirada del otro. La imagen de España en el extranjero. Información comercial española, 722 , 11-25.

Laso, G., \& Justo, P. (2009 ). La imagen de España en China. Boletin económico de ICE, Información Comercial Española, 2972, 95-110. 
Liu, Y. (s.f.). Yang Liu design. Recuperado de

http://sociologiac.net/2008/02/24/conceptualizacion-iconografica-culturaalemana-vs-cultura-china/

Lobato, T. (s.f.). Estereotipos, clichés y competencia intercultural. Propuestas para el aula de ELE- Recuperado de http://cvc.cervantes.es/Ensenanza/Biblioteca_Ele/publicaciones_centros/PDF/br uselas_2013/09_lobato.pdf

López, C. (2005). El componente cultural en la enseñanza de ELE a través de los medios de comunicación y su aplicación en el aula (Memoria de máster). Universidad de Salamanca, Salamanca.

Ministerio de Educación Cultura y Deporte. (s.f). Página oficial del MECD. El mundo estudia español. Recuperado de http://www.mecd.gob.es/dctm/redele/Material-RedEle/el-mundo-estudiaespanol/el-mundo-estudia-espanol2014.pdf?documentId=0901e72b81c71bd2

Miquel, L. (2004). La subcompetencia sociocultural . En J. Sánchez, J.y Santos I. (Eds.). Vademecum para la formación de profesores, Madrid: Sgel. 511-531

Miquel, L. (1999). El choque cultural: reflexiones y recursos para el trabajo en el aula. Carabela, 45, 27-46.

Miquel, L. (2004). Lengua y cultura desde una perspectiva pragmática. RedELE , 2, 137-161.

Miquel, L., \& Sans, N. (2004). El componente cultural: un ingrediente mas en las clases de lengua. RedELE. Revista electronica de didactica ELE, $O$.

Mosquera, M. L. (2011). Su fama lo precede: Uso del método Español Moderno en el contexto universitario en China en la actualidad. Suplementos SinoELE , 5, 9-30.

Navarro, J. M., \& Pardo, J. L. (s.f). Filosofía.net. Recuperado de http://www.filosofia.net/materiales/sofiafilia/hf/soff_26.html

Navarro, P. (2009). Cultura con eñe: Cultura, sociocultura e intercultura en la clase de ELE. Tinkuy: Boletín de investigación y debate , 11, 83-93.

Nikleva, D. (2012). La competencia interculutral y el tratamiento de contenidos culturales en manuales de español como lengua extranjera. Revista española de lingüistica aplicada, 25, 165-188.

Nikleva, D. (2009). Los estereotipos en español: su conocimiento,interpretación y aplicación en el marco de la competencia cultural y pragmático-discrusiva (Curso de doctorado). Universidad de Granada.

Noya, F. J. (s.f). Instituto Elcano Anuario Asia-Pacífico. La imagen de España en China . Recuperado de : http://www.anuarioasiapacifico.es/pdf/2007/Cultura2.pdf

Noya, J. (2007 ). La imagen de España en China. Anuario Asia Pacífico, 453-457. 
Preiswerk, R. y Perrot, D. (1979). Etnocentrismo e Historia. México: Nueva Imagen Real Academia Española. (s.f). Diccionario de la lengua española . Recuperado de: http://dle.rae.es/?id=GqSjqfE\&o=h

Real Instituto Elcano. (s.f.). Barómetro de la imagen de España. Resultados diciembre 2014-enero 2015. Recuperado de : http://www.realinstitutoelcano.org/wps/portal/web/rielcano_es/encuesta?WCM_ GLOBAL_CONTEXT=/elcano/elcano_es/observatoriomarcaespana/estudios/res ultados/barometro-imagen-espana-5

Sánchez, A. (2009). Enseñanza y aprendizaje de español como lengua extranjera en China. Retos y posibilidades del enfoque comunicativo. MarcoELE , 8.

Sánchez, R. (2005). El reflejo de la competencia socio-pragmática en materiales de ELE. XVI Congreso Internacional de ASELE. La Competencia Pragmática o la Enseñanza del Español como Lengua Extranjera. Oviedo, España.

Soler-Espiauba, D. (2009). Los contenidos culturales en la enseñanza del español como segunda lengua. XI Jornadas de Estudios de Lingüística. Investigaciones lingüísticas en el siglo XXI, Universidad de Alicante.

Yang, S. (2015). Enfoques culturales de la didáctica de traducción español-chino en China (tesis doctoral). Universidad Autónoma de Barcelona.

Yang, T. (2013). Metodología y manuales en la enseñanza de español a sinohablantes. SinoELE, 8, 17-41.

Zhu, F. F. (2010). Material complementario del manual Español Moderno (Pekín 1999). Suplementos SinoELE, 2, 1-136.

Fecha de recepción: 09/05/2016

Fecha de revisión: 02/06/2016

Fecha de aceptación: 28/07/2016 